\title{
LIOUVILLIAN FIRST INTEGRALS FOR GENERALIZED RICCATI POLYNOMIAL DIFFERENTIAL SYSTEMS
}

\author{
JAUME LLIBRE ${ }^{1}$ AND CLÀUDIA VALLS ${ }^{2}$
}

\begin{abstract}
We characterize the generalized Riccati polynomial differential systems of the form $x^{\prime}=y, y^{\prime}=a(x) y^{2}+b(x) y+c(x)$, where $a(x), b(x)$ and $c(x)$ are arbitrary polynomials that have a Liouvillian first integrals.
\end{abstract}

\section{Introduction And STATEMEnt of the MAin RESUlts}

A classical problem in the qualitative theory of planar differential equations depending on parameters is to characterize the existence or non-existence of first integrals in function of these parameters.

Let $x$ and $y$ be complex variables. We consider the system

$$
x^{\prime}=y, \quad y^{\prime}=a(x) y^{2}+b(x) y+c(x),
$$

where $a(x), b(x)$ and $c(x)$ are $C^{1}$ functions on $x$ and the prime denotes derivative with respect to the time $t$ that can be either real or complex. In fact, if $a(x) c(x) \not \equiv 0$ these systems are called generalized Riccati differential systems, if $a(x) \not \equiv 0$ and $c(x) \equiv 0$ they are linear differential systems, and if $a(x) \equiv 0$ they are generalized Lienard differential systems.

Our interest is on the generalized Riccati polynomial differential systems, i.e. when the functions $a(x), b(x)$ and $c(x)$ are polynomials and we want to study its Liouvillian integrability.

The vector field associated to system (1) is

$$
X=y \frac{\partial}{\partial x}+\left(a(x) y^{2}+b(x) y+c(x)\right) \frac{\partial}{\partial y} .
$$

The main objectives of this paper is to characterize the Liouvillian first integrals of the generalized Ricatti polynomial differential systems.

Let $U \subset \mathbb{C}^{2}$ be an open set. We say that the non-constant function $H: U \rightarrow \mathbb{C}$ is a first integral of the polynomial vector field $X$ on $U$ if $H(x(t), y(t))$ is constant for all values of $t$ for which the solution $(x(t), y(t))$ of $X$ is defined on $U$. Clearly $H$ is a first integral of $X$ on $U$ if and only if $X H=0$ on $U$.

We recall that a Liouvillian first integral is a first integral $H$ which is a Liouvillian function, that is, roughly speaking which can be obtained "by quadratures" of elementary functions. For a precise definition see [4]. The study of the Liouvillian first

2010 Mathematics Subject Classification. Primary 34C05, 34A34, 34C14.

Key words and phrases. Liouvillian first integrals, algebraic invariant curves, Riccati polynomial differential equations. 
integrals is a classical problem of the integrability theory of the differential equations which goes back to Liouville, see for details again [4].

For studyng of the existence of Liouvillian first integrals we need to study the so-called Darboux polynomials and exponential factors of the Riccati polynomial differential systems of the second kind.

Let $h=h(x, y) \in \mathbb{C}[x, y] \backslash \mathbb{C}$. As usual $\mathbb{C}[x, y]$ denotes the ring of all complex polynomials in the variables $x$ and $y$. We say that $h=0$ is an invariant algebraic curve of the vector field $X$ associated to the Riccati polynomial differential system (1) if it satisfies

$$
y \frac{\partial h}{\partial x}+\left(a(x) y^{2}+b(x) y+c(x)\right) \frac{\partial h}{\partial y}=K h,
$$

the polynomial $K=K(x, y) \in \mathbb{C}[x, y]$ is called the cofactor of $h=0$ and has degree at most

$$
n=\max \{2+\operatorname{deg} a(x), 1+\operatorname{deg} b(x), \operatorname{deg} c(x)\}-1 .
$$

When $h=0$ is an invariant algebraic curve we also say that $h$ is a Darboux polynomial of the Riccati polynomial differential system. Note that a polynomial first integral is a Darboux polynomial with zero cofactor.

An exponential factor $E$ of system (1) is a function of the form $E=\exp (g / h) \notin \mathbb{C}$ with $g, h \in \mathbb{C}[x, y]$ satisfying $(g, h)=1$ and

$$
\frac{\partial E}{\partial x} y+\frac{\partial E}{\partial y}\left(a(x) y^{2}+b(x) y+c(x)\right)=L E,
$$

for some polynomial $L=L(x, y)$ of degree at most $n$ given in (2), called the cofactor of $E$.

The existence of exponential factors $\exp (g / h)$ is due to the fact that the multiplicity of the invariant algebraic curve $h=0$ is larger than 1 , for more details see $[1,3]$.

Proposition 1. The following statements hold.

(i) If $E=\exp (g / h)$ is an exponential factor for the polynomial system (1) and $h$ is not a constant polynomial, then $h=0$ is an invariant algebraic curve.

(ii) Eventually $e^{g}$ can be exponential factors, coming from the multiplicity of the infinite invariant straight line.

For a geometrical meaning of the exponential factors and a proof of Proposition 1 see [3].

A non-constant function $R: U \rightarrow \mathbb{C}$ is an integrating factor of the polynomial vector field $X$ on $U$, if one of the following three equivalent conditions holds

$$
\frac{\partial(R P)}{\partial x}=-\frac{\partial(R Q)}{\partial y}, \quad \operatorname{div}(R P, R Q)=0, \quad X R=-R \operatorname{div}(P, Q),
$$

on $U$ where $P=y$ and $Q=a(x) y^{2}+b(x) y+c(x)$. As usual the divergence of the vector field $X$ is given by

$$
\operatorname{div}(P, Q)=\frac{\partial P}{\partial x}+\frac{\partial Q}{\partial y}
$$


In [1] the next result is proved.

Theorem 2. Suppose that the polynomial vector field $X$ of degree $m$ defined in $\mathbb{C}^{2}$ admits $p$ invariant algebraic curves $f_{i}=0$ with cofactors $K_{i}$, for $i=1, \ldots, p$ and $q$ exponential factors $E_{j}=\exp \left(g_{j} / h_{j}\right)$ with cofactors $L_{j}$, for $j=1, \ldots, q$. Then there exist $\lambda_{i}, \mu_{j} \in \mathbb{C}$ not all zero such that

$$
\sum_{i=1}^{p} \lambda_{i} K_{i}+\sum_{j=1}^{q} \mu_{j} L_{j}=-\operatorname{div}(P, Q),
$$

if and only if the function of Darboux type

$$
f_{1}^{\lambda_{1}} \cdots f_{p}^{\lambda_{p}} E_{1}^{\mu_{1}} \cdots E_{q}^{\mu_{q}}
$$

is an integrating factor of the vector field $X$.

The proof of the following result is given in $[2,4]$.

Theorem 3. The polynomial differential system (1) has a Liouvillian first integral if and only if it has an integrating factor of Darboux type.

The following is the first main result of this paper. Its proof follows by direct computations.

Theorem 4. The following holds for the generalized Riccati polynomial differential systems (1):

(a) Assume $b(x) \equiv 0$ then

$$
\frac{y^{2}}{2} \exp \left(-2 \int a(x) d x\right)-\int c(x) \exp \left(-2 \int a(u) d u\right) d x
$$

is a Liouvillian first integral.

(b) Assume $b(x) \not \equiv 0, c(x)=\kappa a(x)$ and $b(x)=\kappa_{1} a(x)$ with $\kappa, \kappa_{1} \in \mathbb{C}$, then

(b.1) if $\kappa=\kappa_{1}^{2} / 4$ we have that

$$
\int a(x) d x-\frac{\kappa_{1}}{\kappa_{1}+2 y}-\log \left(\kappa_{1}+2 y\right)
$$

is a Liouvillian first integral;

(b.2) if $\kappa \neq \kappa_{1}^{2} / 4$ we have that

$$
\int a(x) d x-\frac{1}{2} \log \left(y^{2}+\kappa_{1} y+\kappa\right)+\frac{\kappa_{1}}{\sqrt{4 \kappa-\kappa_{1}^{2}}} \arctan \left(\frac{\kappa_{1}+2 y}{\sqrt{4 \kappa-\kappa_{1}^{2}}}\right)
$$

is a Liouvillian first integral.

From now on we consider the case in which $b(x) \not \equiv 0$ and $c(x) / a(x) \notin \mathbb{C}$, or $b(x) \not \equiv 0$ and $b(x) / a(x) \notin \mathbb{C}$.

Theorem 5. The generalized Riccati polynomial differential systems (1) with either $b(x) \not \equiv 0$ and $c(x) / a(x) \notin \mathbb{C}$, or $b(x) \not \equiv 0$ and $b(x) / a(x) \notin \mathbb{C}$ have no Liouvillian first integrals.

The proof of Theorem 5 is given in Section 2 . 


\section{Proof of TheOrem 5}

For proving Theorem 5 we first characterize the Darboux polynomials (either with zero or with nonzero cofactor) of the generalized Ricatti polynomial differential systems.

Theorem 6. The generalized Riccati polynomial differential systems (1) with either $b(x) \not \equiv 0$ and $c(x) / a(x) \notin \mathbb{C}$, or $b(x) \not \equiv 0$ and $b(x) / a(x) \notin \mathbb{C}$, have no Darboux polynomials.

We separate the proof of Theorem 6 in several steps.

Lemma 7. The generalized Riccati polynomial differential systems (1) have no polynomial first integrals.

Proof. We proceed by contradiction. Let $H$ be a polynomial first integral of system (1), that is

$$
y \frac{\partial H}{\partial x}+\left(a(x) y^{2}+b(x) y+c(x)\right) \frac{\partial H}{\partial y}=0 .
$$

We write $H$ as a polynomial in the variable $y$, i.e.

$$
H(x, y)=\sum_{j=0}^{m} h_{j}(x) y^{j}, \quad \text { where } h_{j}(x) \text { is a polynomial in the variable } x \text {. }
$$

Without loss of generality we can assume that $h_{m}(x) \neq 0$.

Computing the coefficient of degree $m+1$ in the variable $y$ in (3) we get that

$$
h_{m}^{\prime}(x)+m a(x) h_{m}(x)=0, \quad \text { that is } \quad h_{m}(x)=C \exp \left(-m \int a(x) d x\right) \neq 0 .
$$

Since $h_{m}(x)$ is a polynomial, we must have $m=0$ because $a(x) \not \equiv 0$. Then $H=h_{0}(x)$. In view of $(3)$ we get that $H$ satisfies

$$
H^{\prime}(x)=0, \quad \text { that is } H(x) \in \mathbb{C},
$$

a contradiction with the fact that $H$ is a polynomial first integral.

Lemma 7 states that the generalized Riccati polynomial differential systems (1) have no Darboux polynomials with zero cofactor.

The proof of the following proposition is well-known and can be found in [1].

Proposition 8. We suppose that $h \in \mathbb{C}[x, y]$ and let $h=h_{1}^{n_{1}} \cdots h_{r}^{n_{r}}$ be its factorization in irreducible factors over $\mathbb{C}[x, y]$. Then for a polynomial system (1) $h=0$ is an invariant algebraic curve with cofactor $K_{h}$ if and only if $h_{i}=0$ is an invariant algebraic curve for each $i=1, \ldots, r$ with cofactor $K_{h_{i}}$. Moreover $K=n_{1} K_{h_{1}}+\cdots+n_{r} K_{h_{r}}$.

In view of Proposition 8 to study the Darboux polynomials with non-zero cofactor it is enough to study the irreducible ones.

Lemma 9. Let $h=h(x, y)$ be an irreducible Darboux polynomial of the generalized Riccati polynomial differential system (1) with cofactor $K \neq 0$. Then $K=m b(x)+$ $n^{\prime}(x)-n(x) a(x)+m a(x) y$ with $m$ a non-negative integer and $n \in \mathbb{C}[x]$. 
Proof. The cofactor $K$ of any irreducible Darboux polynomial of the generalized Riccati polynomial differential system (1), has degree at most $n$ (see (2)). We write it as $K(x, y)=\sum_{j=0}^{n} K_{j}(x) y^{j}$, where $K_{j}=K_{j}(x)$ is a polynomial in the variable $x$ and has at most degree $n-j$. Since $h$ is a Darboux polynomial of system (1) with cofactor $K$ it satisfies

$$
y \frac{\partial h}{\partial x}+\left(a(x) y^{2}+b(x) y+c(x)\right) \frac{\partial h}{\partial y}=\left(\sum_{j=0}^{n} K_{j}(x) y^{j}\right) h .
$$

We write $h$ as a polynomial in the variable $y$, i.e. $h(x, y)=\sum_{j=0}^{m} h_{j}(x) y^{j}$, where each $h_{j}(x)$ is a polynomial in the variable $x$. Without loss of generality we can assume that $h_{m}(x) \neq 0$.

Assume $n \geq 2$. Computing the coefficient of $y^{n+m}$ in (4) we get

$$
0=K_{n}(x) h_{m}(x) \text { that is } K_{n}(x)=0 .
$$

So $n \in\{0,1\}$ and consequently $K=K_{0}(x)+y K_{1}(x)$.

Now computing the coefficient of $y^{m+1}$ in (4) we get

$$
h_{m}^{\prime}(x)+m a(x) h_{m}(x)=h_{m}(x) K_{1}(x),
$$

that is $h_{m}^{\prime}(x)+\left(m a(x)-K_{1}(x)\right) h_{m}(x)=0$. Hence, $h_{m}(x)=C \exp \left(-\int(m a(x)-\right.$ $\left.K_{1}(x)\right) d x$ ) with $C \in \mathbb{C} \backslash\{0\}$. Using that $h_{m}(x) \neq 0$ and that it must be a polynomial, we have $K_{1}(x)=m a(x)$ with $m$ a non-negative integer and $h_{m}(x)=C$. Now, computing the coefficient of $y^{m}$ in (4) we get

$$
h_{m-1}^{\prime}(x)+(m-1) a(x) h_{m-1}(x)+m b(x) h_{m}(x)=m a(x) h_{m-1}(x)+K_{0}(x) h_{m}(x) .
$$

Then since $h_{m}(x)=C$,

$$
h_{m-1}^{\prime}(x)-a(x) h_{m-1}(x)=C\left(K_{0}(x)-m b(x)\right) .
$$

Therefore we have a linear system. Solving it we get

$$
h_{m-1}(x)=C_{1} e^{\int a(x) d x}+C e^{\int a(x) d x} \int\left(K_{0}(x)-m b(x)\right) e^{-\int a(u) d u} d x .
$$

Since $h_{m-1}(x) \in \mathbb{C}[x]$, we deduce that $C_{1}=0$ and

$$
K_{0}(x)-m b(x)=n^{\prime}(x)-n(x) a(x),
$$

with $n \in \mathbb{C}[x]$ and $h_{m-1}(x)=C n(x)$. This completes the proof.

Proposition 10. The generalized Riccati polynomial differential systems (1) with either $b(x) \not \equiv 0$ and $c(x) / a(x) \notin \mathbb{C}$, or $b(x) \not \equiv 0$ and $b(x) / a(x) \notin \mathbb{C}$ have no irreducible Darboux polynomials with non-zero cofactor $K$.

Proof. We write the generalized Riccati polynomial differential system (1) as the differential equation

$$
y \frac{d y}{d x}=a(x) y^{2}+b(x) y+c(x), \quad y=y(x) .
$$


Then, using Lemma 9 the Darboux polynomial $h=h(x, y)=h(x, y(x))$ satisfies $y \frac{d h}{d x}=y \frac{\partial h}{\partial x}+\frac{\partial h}{\partial y}\left(a(x) y^{2}+b(x) y+c(x)\right)=\left(m b(x)+n^{\prime}(x)-n(x) a(x)+m a(x) y\right) h$, where $m$ is a non-negative integer, or equivalently

$$
\log h=K+\int \frac{m b(x)+n^{\prime}(x)-n(x) a(x)+m a(x) y}{y} d x, \quad \text { where } \quad K \in \mathbb{C} .
$$

Hence

(6)

$h=h(x, y(x))=C \exp \left(\int \frac{m b(x)+n^{\prime}(x)-n(x) a(x)+m a(x) y}{y} d x\right), \quad C \in \mathbb{C} \backslash\{0\}$.

Now we write

(7) $a(x) y^{2}+b(x) y+c(x)=\left(y+\Gamma_{1}(x)\right)\left(a(x) y+\Gamma_{2}(x)\right)=\left(a(x) y+\tilde{\Gamma}_{1}(x)\right)\left(y+\tilde{\Gamma}_{2}(x)\right)$, where

(8) $\Gamma_{1}(x)=\frac{b(x)}{2 a(x)}-\frac{\sqrt{b(x)^{2}-4 a(x) c(x)}}{2 a(x)}, \quad \Gamma_{2}(x)=\frac{b(x)}{2}+\frac{\sqrt{b(x)^{2}-4 a(x) c(x)}}{2}$, and $\tilde{\Gamma}_{1}(x)=a(x) \Gamma_{1}(x), \tilde{\Gamma}_{2}(x)=\Gamma_{2}(x) / a(x)$. We consider different cases.

Case 1: $\Gamma_{1}(x)=\kappa \in \mathbb{C}$. In this case, from (8) we have that

$$
\frac{b(x)}{2 a(x)}-\frac{\sqrt{b(x)^{2}-4 a(x) c(x)}}{2 a(x)}=\kappa, \quad \text { i.e. } \quad b(x)-\sqrt{b(x)^{2}-4 a(x) c(x)}=2 \kappa a(x),
$$

which yields

$$
c(x)=\kappa(b(x)-\kappa a(x)) .
$$

Then, again from (8) we get

$$
\Gamma_{2}(x)=b(x)-\kappa a(x) .
$$

Hence, it follows from (5) and (7) that

$y \frac{d y}{d x}=(y+\kappa)\left(a(x) y+\Gamma_{2}(x)\right), \quad$ that is $\frac{a(x) y+\Gamma_{2}(x)}{y}=\frac{d y / d x}{y+\kappa}=\frac{d}{d x} \log (y+\kappa)$,

which implies that (6) becomes

$$
\begin{aligned}
h & =C \exp \left(\int \frac{m b(x)+n^{\prime}(x)-n(x) a(x)-m \Gamma_{2}(x)+m\left(a(x) y+\Gamma_{2}(x)\right)}{y} d x\right) \\
& =C \exp \left(\int\left(\frac{m b(x)+n^{\prime}(x)-n(x) a(x)-m \Gamma_{2}(x)}{y}+m \frac{d}{d x} \log (y+\kappa)\right) d x\right) \\
& =C(y+\kappa)^{m} \exp \left(\int \frac{n^{\prime}(x)-n(x) a(x)+m \kappa a(x)}{y} d x\right),
\end{aligned}
$$

with $C \in \mathbb{C} \backslash\{0\}$. Since $h$ must be a polynomial in the variables $x$ and $y$, we must have

$$
n^{\prime}(x)-n(x) a(x)+m \kappa a(x)=0 .
$$


Solving it we get

$$
n(x)=C_{1} e^{\int a(x) d x}+m \kappa e^{\int a(x) d x} \int a(x) e^{-\int a(u) d u} d x .
$$

Since $n(x)$ is a polynomial we must have $C_{1}=0$ and $m \kappa=0$ and thus $n(x)=0$. If $m=0$ then we have that $K_{0}=K_{1}=0$ and thus $K=0$ a contradiction with the fact that $h$ is a Darboux polynomial with non-zero cofactor. If $\kappa=0$ then $c(x) \equiv 0$ in contradiction with the fact that $c(x) / a(x) \notin \mathbb{C}$. Therefore, this case is not possible.

Case 2: $\Gamma_{1}(x) \notin \mathbb{C}$ and $\tilde{\Gamma}_{2}=\Gamma_{2}(x) / a(x)=\kappa \in \mathbb{C}$. In this case, from (8) we have that

$$
\frac{b(x)}{2 a(x)}+\frac{\sqrt{b(x)^{2}-4 a(x) c(x)}}{2 a(x)}=\kappa, \quad \text { i.e. } \quad b(x)+\sqrt{b(x)^{2}-4 a(x) c(x)}=2 \kappa a(x),
$$

which yields

$$
c(x)=\kappa(b(x)-\kappa a(x)) \text { and } b(x)=2 \kappa a(x),
$$

which is not possible because then we have $\Gamma_{1}(x)=\kappa \in \mathbb{C}$.

Case 3: $\Gamma_{1}(x) \notin \mathbb{C}$ and $\tilde{\Gamma}_{2}=\Gamma_{2}(x) / a(x) \notin \mathbb{C}$. In this case, from (8) we can write

$$
\frac{a(x) y+\Gamma_{2}(x)}{y}=\frac{d y / d x}{y+\Gamma_{1}(x)}=\frac{d}{d x} \log \left(y+\Gamma_{1}(x)\right)-\frac{d \Gamma_{1} / d x}{y+\Gamma_{1}(x)}
$$

and analogously,

$$
\left.\frac{a(x) y+\tilde{\Gamma}_{1}(x)}{y}=\frac{d y / d x}{y+\tilde{\Gamma}_{2}(x)}=\frac{d}{d x} \log \left(y+\tilde{\Gamma}_{2}\right)\right)-\frac{d \tilde{\Gamma}_{2} / d x}{y+\tilde{\Gamma}_{2}(x)} .
$$

In the first case, from (6), we have that

$$
\begin{aligned}
h & =C\left(y+\Gamma_{1}(x)\right)^{m} \exp \left(\int \frac{m b(x)+n^{\prime}(x)-n(x) a(x)-m \Gamma_{2}(x)}{y} d x\right) \\
& \exp \left(-m \int \frac{d \Gamma_{1} / d x}{y+\Gamma_{1}(x)} d x\right),
\end{aligned}
$$

which to be a polynomial in the variables $x$ and $y$ we must have

$$
\frac{m b(x)+n^{\prime}(x)-n(x) a(x)-m \Gamma_{2}(x)}{y}=m \frac{d \Gamma_{1} / d x}{y+\Gamma_{1}(x)},
$$

or equivalently,

$$
\begin{aligned}
& m b(x)+n^{\prime}(x)-n(x) a(x)-m \Gamma_{2}(x)=m \frac{d \Gamma_{1}}{d x}, \\
& \Gamma_{1}(x)\left(m b(x)+n^{\prime}(x)-n(x) a(x)-m \Gamma_{2}(x)\right)=0 .
\end{aligned}
$$

By hypothesis we have that $\Gamma_{1}(x) \neq 0$ and thus $m b(x)+n^{\prime}(x)-n(x) a(x)-m \Gamma_{2}(x)=$ 0 , but then from the first relation we have $d \Gamma_{1} / d x=0$, which again is not possible because $\Gamma_{1}(x) \notin \mathbb{C}$. Hence this case is not possible. 
In the second case we have

$$
\begin{aligned}
& h=C\left(y+\tilde{\Gamma}_{2}(x)\right)^{m} \exp \left(\int \frac{m b(x)+n^{\prime}(x)-n(x) a(x)-m \tilde{\Gamma}_{1}(x)}{y} d x\right) \\
& \quad \exp \left(-m \int \frac{d \tilde{\Gamma}_{2} / d x}{y+\tilde{\Gamma}_{2}(x)} d x\right),
\end{aligned}
$$

which again is never a polynomial in the variables $x$ and $y$. Hence this case is never possible. This completes the proof of the proposition.

Proof of Theorem 6. The proof of Theorem 6 is an immediate consequence of Lemma 7 and Proposition 10.

Lemma 11. Assume that $\exp \left(g_{1} / h_{1}\right), \ldots, \exp \left(g_{r} / h_{r}\right)$ are exponential factors of some polynomial differential system

$$
x^{\prime}=P(x, y), \quad y^{\prime}=Q(x, y), \quad P, Q \in \mathbb{C}[x, y],
$$

with cofactors $L_{j}$ for $j=1, \ldots, r$. Then $\exp (G)=\exp \left(g_{1} / h_{1}+\cdots+g_{r} / h_{r}\right)$ is also an exponential factor of system (9) with cofactor $L=\sum_{j=1}^{r} L_{j}$.

Proof. Using that for $j=1, \ldots, r, E_{1}=\exp \left(g_{1} / h_{1}\right), \ldots, E_{r}=\exp \left(g_{r} / h_{r}\right)$ are exponential factors of system (9) with cofactors $L_{j}$ we have

$$
\frac{\partial\left(g_{j} / h_{j}\right)}{\partial x} P(x, y) E_{j}+\frac{\partial\left(g_{j} / h_{j}\right)}{\partial y} Q(x, y) E_{j}=L_{j} E_{j},
$$

or equivalently,

$$
\frac{\partial\left(g_{j} / h_{j}\right)}{\partial x} P(x, y)+\frac{\partial\left(g_{j} / h_{j}\right)}{\partial y} Q(x, y)=L_{j}
$$

Therefore if we set $G=\sum_{j=1}^{r} g_{j} / h_{j}$ we get that

$$
\frac{\partial G}{\partial x} P(x, y)+\frac{\partial G}{\partial y} Q(x, y)=\sum_{j=1}^{r} L_{j}=L,
$$

and thus if $E=\exp (G)$ we obtain

$$
\frac{\partial G}{\partial x} P(x, y) E+\frac{\partial G}{\partial y} Q(x, y) E=L E .
$$

This concludes the proof of the lemma.

Proof of Theorem 5. It follows from Theorem 6 that the generalized Riccati polynomial differential system (1) has no Darboux polynomials. Hence, it follows from Proposition 1 and Theorems 2 and 3 that to have a Liouvillian first integral we must have $q$ exponential factors $E_{j}=\exp \left(g_{j}\right)$ with cofactors $L_{j}$ such that

$$
\sum_{j=1}^{q} \mu_{j} L_{j}=-2 a(x) x-b(x) .
$$


Let $G=\sum_{j=1}^{q} \mu_{j} g_{j} \in \mathbb{C}[x, y]$. Then $E=\exp (G)=\exp \left(\sum_{j=1}^{q} \mu_{j} g_{j}\right)$, is an exponential factor of system (1) with the cofactor $L=\sum_{j=1}^{q} \mu_{j} L_{j}$ (see Lemma 11) and $E$ satisfies

$$
y \frac{\partial E}{\partial x}+\frac{\partial E}{\partial y}\left(a(x) y^{2}+b(x) y+c(x)\right)=L E,
$$

that is

$$
y \frac{\partial G}{\partial x}+\frac{\partial G}{\partial y}\left(a(x) y^{2}+b(x) y+c(x)\right)=L=-2 a(x) y-b(x) .
$$

We write $G$ as a polynomial in the variable $y$ as follows

$$
G=\sum_{j=0}^{m} G_{j}(x) y^{j} .
$$

Computing the coefficient of $y^{m+1}$ with $m \geq 1$ in (10) we get

$$
G_{m}^{\prime}(x)+\operatorname{ma}(x) G_{m}(x)=0,
$$

that is

$$
G_{m}(x)=C_{m} \exp \left(-m \int a(x) d x\right), \quad C_{m} \in \mathbb{C} .
$$

Since $G_{m}$ must be a polymoimal we must have $G_{m}(x)=0$ and thus $G=G_{0}(x)$. Then introducing it in (10) we obtain

$$
y G_{0}^{\prime}(x)=-2 a(x) y-b(x),
$$

and since $b(x) \not \equiv 0$ we get that this case is not possible. This concludes the proof.

\section{ACKNowledgements}

The first author is partially supported by the MICINN/FEDER grant MTM2008 03437, AGAUR grant 2009SGR-410 and ICREA Academia. The second author has been partially supported by FCT through CAMGDS, Lisbon.

\section{REFERENCES}

[1] C. Christopher And J. Llibre, Integrability via invariant algebraic curves for planar polynomial differential sysetms, Ann. Diff. Equations 16 (2000), 5-19.

[2] C. Christopher, Liouvillian first integrals of second order polynomial differential equations, Electron J. Differential Equations 49 (1999), 7 pp.

[3] C. Christopher, J. Llibre and J.V. Pereira, Multiplicity of invariant algebraic curves in polynomial vector fields, Pacific J. Math. 229 (2007), 63-117.

[4] M.F. Singer, Liouvillian solutions of nth order homogeneous linear differential equations, Amer. J. Math. 103 (1981), 661-682.

1 Departament de Matemàtiques, Universitat Autònoma de Barcelona, 08193 Bellaterra, Barcelona, Catalonia, Spain

E-mail address: jllibre@mat.uab.cat

${ }^{3}$ Departamento de Matemática, Instituto Superior Técnico, Universidade Técnica De Lisboa, Av. Rovisco Pais 1049-001, Lisboa, Portugal

E-mail address: cvalls@math.ist.utl.pt 